Bull. Egypt. Soc. Physiol. Sci. Vol. (41), Issue (4), 525-536

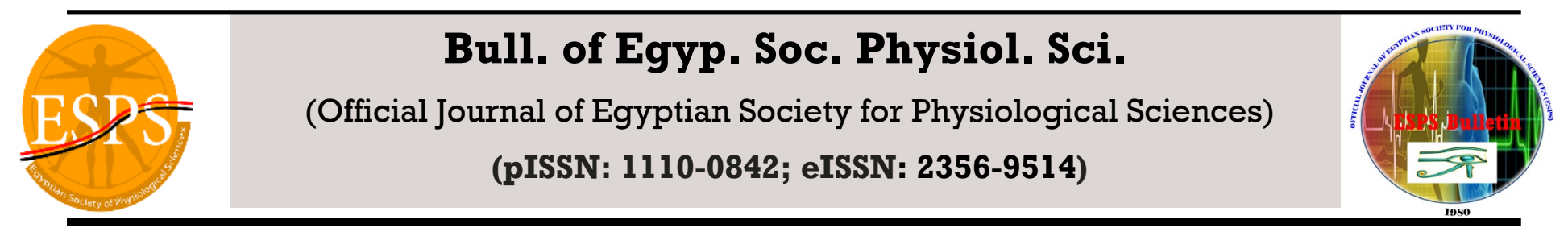

\title{
Replenishing glutathione counters ciprofloxacin-induced acute liver failure via possible gene modifying mechanism.
}

\author{
Yahya M. Naguib" ${ }^{1,2}$, Shaimaa M. Motawea² and Omnia Ameen* \\ ${ }^{1}$ Physiology Department, College of Medicine and Medical Sciences, Arabian Gulf University, Manama, Bahrain \\ ${ }^{2}$ Clinical Physiology Department, Faculty of Medicine, Menoufia University, Menoufia, Egypt
}

Submit Date: Feb 15, 2021

Revise Date: March 14, 2021

Accept Date: March 28, 2021

Keywords

- Acute liver failure

- $\quad$ oxidative stress

- glutathione

- Ciprofloxacin

\begin{abstract}
Background: Acute liver failure (ALF) can be defined as rapid loss of liver functions even in the absence of pre-existing liver disorders. ALF is a medical emergency that necessitates hospitalization and, if not properly treated, a liver transplant may be the only cure. Herein, we studied the potential therapeutic effects of exogenous glutathione on drug-induced ALF. Materials: Thirty male Wistar albino rats were used in the present study. Rats were divided (10/group) into: control group (received only intra-peritoneal injection of distilled water), ciprofloxacin-treated group (received intra-peritoneal injection of $800 \mathrm{mg} / \mathrm{kg} / \mathrm{day}$ ciprofloxacin for 15 consequent days with concomitant intra-peritoneal injection of distilled water), and ciprofloxacin + glutathione-treated group (received intra-peritoneal injection of $800 \mathrm{mg} / \mathrm{kg} /$ day ciprofloxacin for 15 consequent days with concomitant intra-peritoneal injection of $200 \mathrm{mg} / \mathrm{kg} /$ day glutathione). Measurement of serum ALT, AST, GGT and LDH enzymes activities were performed, and hepatic gene expression of CYP3A, GPx, GSR and Nrf2 mRNA was assessed. Results: Treatment with ciprofloxacin resulted in significant increase in ALT, AST, GGT and LDH enzymes activities when compared to the control group. Ciprofloxacin also induced significant down-regulation of CYP3A, GPx, GSR and Nrf2 mRNA gene expression when compared to the corresponding values in the control group. Glutathione administration significantly normalized ALT, AST, GGT and LDH enzymes activities and up-regulated CYP3A, GPx, GSR and Nrf2 mRNA gene expression. Conclusion: Glutathione could play potential therapeutic roles in the treatment of acute liver failure by preventing oxidative stress-induced disruption of hepatocyes cell membranes and alteration in cytochrome $\mathrm{P} 450$ and the antioxidant genes.
\end{abstract}

Corresponding author: Omnia Ameen Clinical Physiology Department, Faculty of Medicine, Menoufia University, Menoufia, Egypt. Tel: +20482222731, Fax: +20482317508, e-mail: omnia.ameen@med.menofia.edu.eg. 


\section{INTRODUCTION}

The liver plays a wide array of vital functions in order to maintain homeostasis. Acute liver failure (ALF) is a complex ,and often catastrophic, condition manifesting as altered mental status due to rapid decline of liver function without preexisting liver disease (1). Drugs account for over $50 \%$ of acute liver failure cases followed by viral hepatitis (2). Antibiotics are the drugs class most frequently associated with idiosyncratic druginduced liver injury (3).

Liver is a major organ attacked by reactive oxygen species (ROS). Kupffer cells, hepatic stellate cells and endothelial cells are potentially more sensitive to oxidative stress-related molecules (4). A variety of cytokines such as tumor necrosis factor (TNF)- $\alpha$ can be produced in Kupffer cells in response to oxidative stress, which may boost inflammation and apoptosis (5). Oxidative stress not only triggers hepatic damage by inducing irreversible damage of lipids, proteins and DNA, but it also plays a role in altering pathways that control biological functions. Ever since these pathways regulate gene transcription, protein expression, cell apoptosis and hepatic stellate cell activation; oxidative stress is considered as one of the most vital pathophysiological mechanisms that underlie the initiation and progression of various liver diseases (6).

Fluoroquinolones are a series of synthetic antibacterial agents used for the treatment of bacterial infections. Ciprofloxacin, an active quinolone carboxylic acid, is a widely prescribed fluoroquinolone that has broad antimicrobial coverage and high oral bioavailability (7). Ciprofloxacin-induced acute hepatitis resembles in many aspects symptoms of non-drug-induced acute hepatitis (8). Fluoroquinolones-induced hepatotoxicity was marked by elevated levels of alanine aminotransferase (ALT), aspartate aminotransferase (AST), alkaline phosphatase, direct and total bilirubin, and prolonged prothrombin time (9). Fluoroquinolones-induced hepatotoxicity has been suggested as a result of the generation of oxidative radicals with subsequent liver damage (10).

Antioxidants provide a promising therapeutic strategy to prevent and cure liver diseases. In vivo animal studies demonstrated the potentiality of antioxidants in protecting the liver from oxidative stress insults (11). Glutathione (GSH), a cysteineglutamic acid-glycine tripeptide, is the most abundant hepatic antioxidant. GSH is synthesized by the hepatocytes, thereby, protecting the liver from oxidative stress. GSH serves as a cofactor for the enzyme glutathione peroxidase (GPx) in metabolizing hydrogen and lipid peroxides (12). GSH may conjugate to electrophilic endogenous compounds and xenobiotics via glutathione Stransferase (GST) family of enzymes, allowing safe and efficient elimination from the body (13). Moreover, GSH is able to regenerate other antioxidants such as vitamins $\mathrm{C}$ and $\mathrm{E}$ back to their active forms (14). Disruption of GSH homeostasis is evident in chronic alcoholic liver disease, hepatitis $\mathrm{C}$ infection, hypoxic/ischemic and/or reperfusion injury, and non-alcoholic fatty liver disease (15). Several studies have shown the therapeutic efficiency of oral or intravenous GSH administration in paracetamol-induced liver injury, alcoholic liver cirrhosis and chronic fatty liver diseases $(16,17)$. 
A family of transcription factors, including the nuclear factor erythroid 2 (NFE2)-related factor (Nrf2), controls the biosynthesis of many of the naturally occurring antioxidants (18). Nrf proteins bind to a specific DNA sequence; the antioxidant response element (ARE) which could be found in the promoters of many chemoprotective genes including those involved in the response to oxidative stress. Binding of Nrf to ARE enhances the transcription of many antioxidants' genes including GPx and GST (18, 19). The cytochrome P450 (CYPs 1-4) enzymes family are highly expressed in mammalian hepatocytes and strongly linked to pharmaceutical metabolism. Precisely, CYP3A4, CYP2D6, CYP2C9 and CYP1A2 might be responsible for $50 \%, 25 \%, \quad 15 \%$ and $5 \%$ of human drug metabolism respectively (20). It is during the direct redox cycling of an agent, or its metabolism by cytochrome $\mathrm{P} 450$ where the generation of free radicals occurs (21).

Herein, we tested the therapeutic effects of glutathione on ciprofloxacin-induced acute liver injury. We also explored the possible underlying molecular mechanisms.

\section{METHODS}

All experiments were conducted in adherence to the Guiding Principles in the Use and Care of Animals published by the National Institutes of Health (NIH Publication No 85-23, Revised 1996). Animal care and use were approved by the Faculty of Medicine Menoufia University Ethics Committee.

\section{Animals and experimental design}

Thirty male Wistar rats weighing 150-200 g were purchased from a local laboratory animal providing facility. Rats were acclimatized for 10 days before the start of experiments. Rats were given free access to normal chow diet and water in an air-conditioned room with a 12 hour light-dark cycles. At the end of the study, all rats were scarified by cervical dislocation.

After acclimatization, rats were randomly divided (10 rats per group) into the following 3 groups:

1- Control group: received intra-peritoneal injection of distilled water for 15 consecutive days.

2- Ciprofloxacin (Cipro)-treated group: received concomitantly intra-peritoneal injection of $800 \mathrm{mg} / \mathrm{kg} /$ day ciprofloxacin (Ciproxin solution for infusion, Bayer plc, Reading Berkshire, United Kingdom) for 15 consecutive days plus intra-peritoneal injection of distilled water.

3- Ciprofloxacin + glutathione (Cipro + Glut)-treated group: received concomitantly intra-peritoneal injection of $800 \mathrm{mg} / \mathrm{kg} /$ day ciprofloxacin for 15 consecutive days plus intra-peritoneal injection of $200 \mathrm{mg} / \mathrm{kg} /$ day glutathione (Advanced Glutathione, Rex Pharma Group, Budapest, Hungary).

\section{Blood and liver samples collection}

After 15 days, rats were fasted overnight after the last injection and anaesthetised by sodium thiopental (STP, $60 \quad \mathrm{mg} / \mathrm{kg}$ intraperitoneal injection). Blood was collected via cardiac puncture. Blood samples were allowed to coagulate for 30 minutes at room temperature, and were then centrifuged at $3000 \mathrm{rpm}$ for $15 \mathrm{~min}$. The serum was separated, collected and samples were stored at $-20{ }^{\circ} \mathrm{C}$ for further investigations. The liver was removed and stored at -80 or freshly used for 
real time quantitative reverse transcriptionpolymerase chain reaction (RT-PCR) experiments.

Biochemical analysis

Serum levels of alanine aminotransferase (ALT), aspartate aminotransferase (AST), lactate dehydrogenase (LDH) and gamma-glutamyl transferase (GGT) (MyBioSource Inc, San Diego, CA, USA) enzymes activities were determined by ELISA technique using an automatic optical reader (SUNRISE Touchscreen, TECHAN, Salzburg, Austria).

Analysis of gene expression quantitative RT-PCR $(q R T-P C R)$

Gene specific primers were designed using Primer Express Software version $2.0 \quad$ (Applied Biosystems, USA). Glyceraldehyde 3-phosphate dehydrogenase (GAPDH) was used as the housekeeping control gene (Table 1). RT- PCR assays were performed in duplicate for all target and housekeeping genes using Applied Biosystems 7500 FAST 96-well PCR machine (USA). To evaluate the effects of glutathione on ciprofloxacin-induced ALF, the mRNA expression levels of cytochrome P450-family 3- subfamily A (CYP3A), glutathione peroxidise (GPx), glutathione reductase (GSR) and nuclear factor (erythroid-derived 2)-like 2 (Nrf2) genes were evaluated by RT-PCR assay as described previously (22-24). Total RNA was extracted following homogenizing frozen or fresh liver specimens using TRI reagent (Sigma-Aldrich, UK). Reverse transcription of hepatic RNA was performed using high-capacity RNA-to-cDNA kit (Applied Biosystems, CA, USA). Afterwards, the generated cDNA was used to measure mRNA expression for the target genes. With GAPDH serving as the endogenous housekeeping gene control, relative mRNA expression of the target gene of interest was calculated using the comparative $\mathrm{Ct}(2-\Delta \mathrm{Ct})$ method. Data were expressed as a ratio (target gene/GAPDH) and were shown as the mean \pm standard error of mean of at least three independent experiments.

\section{Statistical analysis}

Analyses of Variances (ANOVA) with Tukey's post hoc tests were used for statistical analysis of the data using Origin ${ }^{\circledR}$ software. Results are expressed as mean \pm standard error (SE), and $\mathrm{p}$ values $<0.05$ were considered significant.

\section{RESULTS}

The serum levels of the liver enzymes ALT, AST, GGT and LDH were significantly higher $(\mathrm{P}<0.05)$ in the ciprofloxacin treated rats $(88.96 \pm 5.58$, $130.29 \pm 19.93,87.72 \pm 12.53$, and $5.28 \pm 1.01 \mathrm{IU} / 1$ respectively), when compared to the corresponding values in the control group $(4.48 \pm 0.75,3.47 \pm 0.30$, $1.97 \pm 0.49$ and, $0.41 \pm 0.14 \quad \mathrm{IU} / 1$ respectively). Treatment with glutathione resulted in significant decrease $(\mathrm{P}<0.05)$ in the mean values of ALT, AST, LDH and GGT enzymes $(4.56 \pm 0.35$, $3.18 \pm 0.22, \quad 2.47 \pm 0.32$ and $0.50 \pm 0.21 \quad$ IU/1 respectively) in the ciprofloxacin + glutathione treated group when compared to the corresponding values in the ciprofloxacin treated group. There was no significant difference $(\mathrm{P}>0.05)$ when comparing the corresponding values in the ciprofloxacin + glutathione and control groups (Figure 1).

The mRNA gene expression of the CYP3A, GPx, GSR and Nrf2 proteins in the ciprofloxacin treated group $\quad(0.54 \pm 0.03, \quad 0.21 \pm 0.02, \quad 0.29 \pm 0.05$ and $0.35 \pm 0.02$ respectively) was significantly downregulated $(\mathrm{P}<0.05)$ when compared to the corresponding values in the control group (1). 
Glutathione administration resulted in significant up-regulation $(\mathrm{P}<0.05)$ of the expression of CYP3A, GPx, GSR and Nrf2 mRNA genes $(2.01 \pm 0.21, \quad 1.69 \pm 0.02, \quad 2.30 \pm 0.17$ and $1.7 \pm 0.1$ respectively), when compared to the corresponding values in the ciprofloxacin treated group. All the genes were significantly up-regulated $(\mathrm{P}<0.05)$ in the ciprofloxacin and glutathione treated group when compared to the corresponding values in the control group (Figure 2).

Table 1: Primers used for measuring the expression of CYP3A, GPx, GSR and Nrf2 genes.

\begin{tabular}{|l|l|l|}
\hline CYP3A & Forward & GAAACTGCAGGAGGAGATCG \\
\hline & Reverse & TCACAGTATCATAGGTGGGAGGT \\
\hline GPx & Forward & AGTTCGGACATCAGGAGAATGGCA \\
\hline & Reverse & TCACCATTCACCTCGCACTTCTCA \\
\hline GSR & Forward & GGAAGTCAACGGGAAGAAGTTCACTG \\
\hline & Reverse & CAATGTAACCGGCACCCACAATAAC \\
\hline Nrf2 & Forward & CACATCCAGACAGACACCAGT \\
\hline & Reverse & CTACAAATGGGAATGTCTCTGC \\
\hline GAPDH & Forward & TGCACCACCAACTGCTTAGC \\
\hline & Reverse & GGCATGGACTGTGGTCATGAG \\
\hline
\end{tabular}
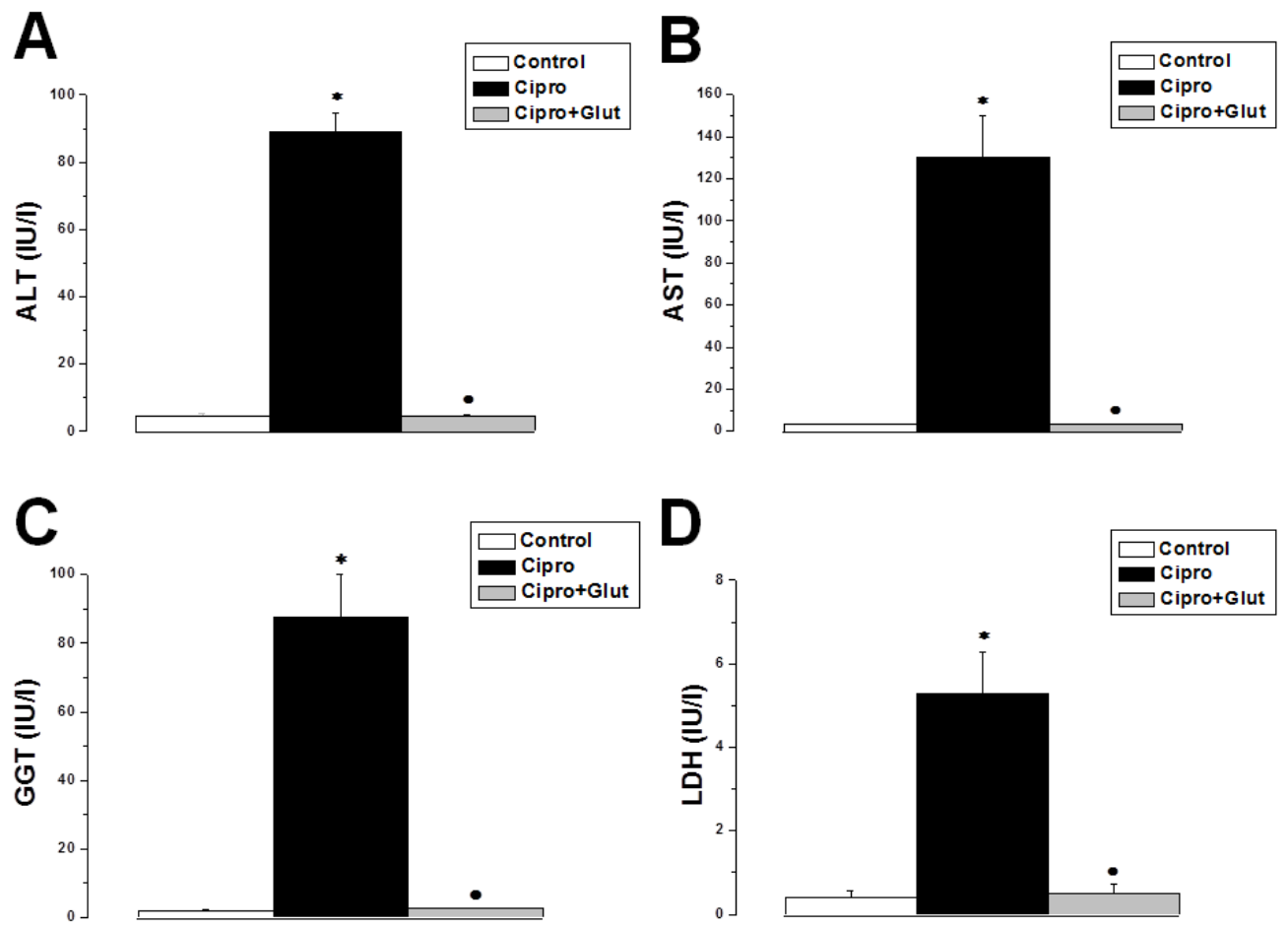

Figure 1: Glutathione normalizes liver enzymes in ciprofloxacin induced acute liver failure. (A) Serum ALT levels in control (white column), ciprofloxacin-treated (black column) and ciprofloxacin + glutathione-treated (light grey column) groups. (B) Serum AST levels in control (white column), ciprofloxacin-treated (black column) and ciprofloxacin + glutathione-treated (light grey column) groups. (C) Serum GGT levels in control (white column), ciprofloxacin-treated (black column) and ciprofloxacin + glutathione-treated (light grey column) groups. (D) Serum LDH levels in control (white column), ciprofloxacin-treated (black column) and ciprofloxacin + glutathione-treated (light grey column) groups. (Significant $=\mathrm{p}<0.05,{ }^{*}$ significant when compared to the control group, $\bullet$ significant when compared to the Ciprotreated group. Number of rats $=10 /$ group). 

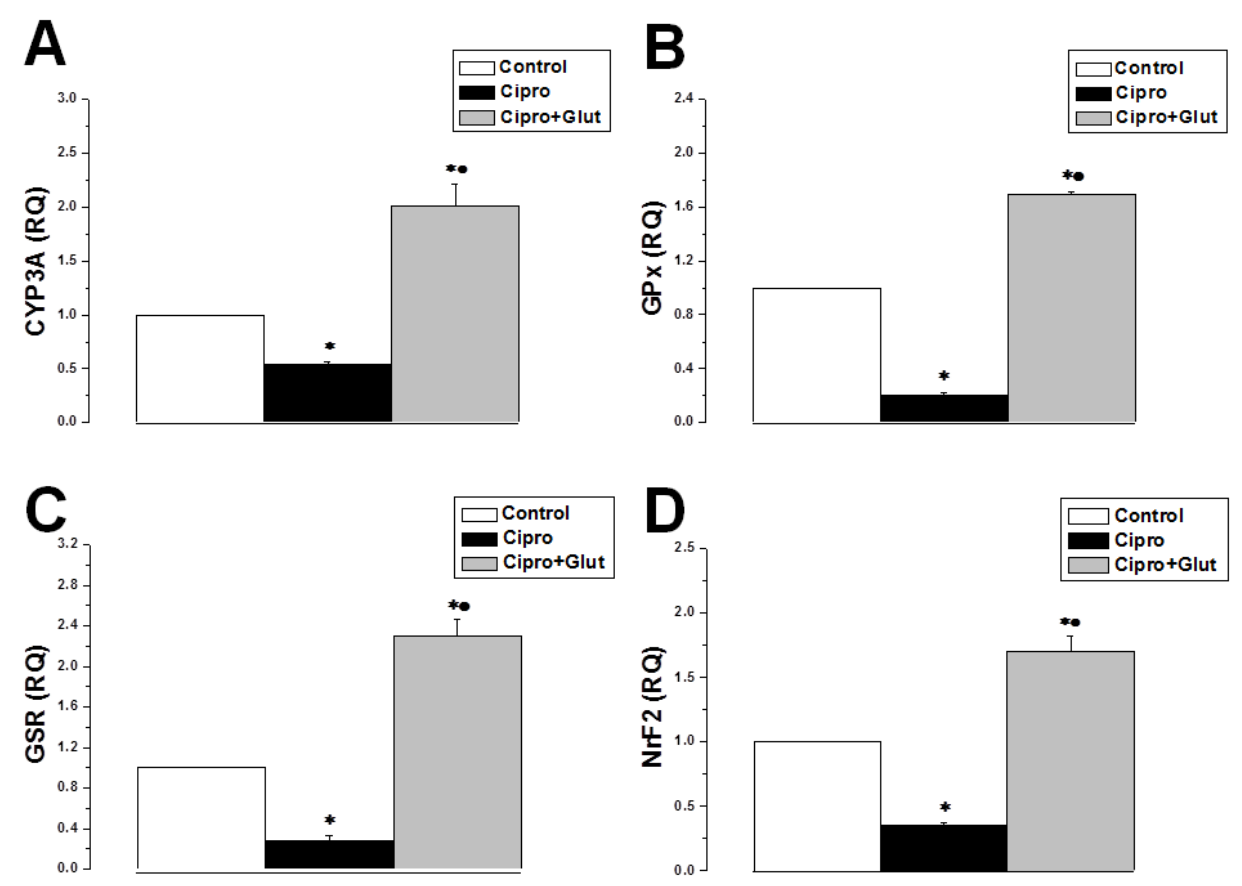

Figure 2: Gene modifying effects of glutathione in ciprofloxacin induced acute liver failure. (A) CYP3A mRNA gene expression levels in control (white column), ciprofloxacin-treated (black column) and ciprofloxacin + glutathionetreated (light grey column) groups. (B) GPx mRNA gene expression levels in control (white column), ciprofloxacintreated (black column) and ciprofloxacin + glutathione-treated (light grey column) groups. (C) GSR mRNA gene expression levels in control (white column), ciprofloxacin-treated (black column) and ciprofloxacin + glutathione-treated (light grey column) groups. (D) Nrf2 mRNA gene expression levels in control (white column), ciprofloxacin-treated (black column) and ciprofloxacin + glutathione-treated (light grey column) groups. (Significant $=p<0.05, *$ significant when compared to the control group, $\bullet$ significant when compared to the Cipro-treated group. Number of rats $=$ 10/group).

\section{DISCUSSION}

Liver remains the principal organ for maintaining homeostasis. Drugs are considered as a central cause of liver failure; the majority of idiosyncratic drug reactions results in deleterious effects ending by even liver transplantation (25). Oxidative stress has been suggested as an underlying mechanism mediating ciprofloxacin-induced ALF. Indeed, oxidant/antioxidant imbalance is the principal signal in the progression of different liver disorders (26). Therefore, synthetic molecules, plant derived compounds, herbal preparations or GSH itself were vastly studied as potential therapeutic strategies for liver diseases. The main aim was to restore the oxidative stress to antioxidants level balance. Above all, the fact that replenishment of GSH, a major determinant of cellular antioxidant-potential, could deliberate the progress severity of various oxidative stressinduced liver insults remains of great interest (26, 27).

In the present study, ciprofloxacin-induced hepatotoxicity was characterized by elevation of liver damage hallmarks; ALT, AST, LDH and GGT. Amino-transferases are the most specific and routinely utilized indicators of hepatocellular necrosis (9). ALT is primarily localized in the liver, while AST is present in a wide range of tissues such as the heart, skeletal muscle, kidney, brain, and the liver. High levels of AST may indicate liver damage, as well as myocardial infarction and muscle injury. Whereas, ALT is 
more specific to liver damage (28). GGT is a membrane-bound glycoprotein enzyme. GGT is mainly derived from hepatocytes, biliary epithelial cells, renal tubules, pancreas and intestine (9). Elevated levels of serum GGT activity has been considered as a sensible marker of hepatic disorders. Interestingly, the biological function of GGT at physiological concentrations mainly lies in the maintenance of the intracellular levels of GSH and the metabolism of GSH conjugates (29). The production of LDH increases under low oxygen concentrations, therefore, $\mathrm{LDH}$ is considered as a hallmark of hepatic hypoxic conditions (30). In our hands, administration of glutathione resulted in restoration of the liver functions profile in the glutathione- treated group. The glutathione system plays a central role as an antioxidant protecting hepatocytes from the detrimental effects of free radicals. In this system, reduced glutathione (GSH) is utilized to detoxify organic and inorganic peroxides via glutathione GPx activity, whereas GSR mediates the reduction of oxidized glutathione (GS) by using NADPH $(26,31)$. It has been reported that ciprofloxacin could induce hepatic oxidative stress with an associated rise in serum ALP, acid phosphatase (ACP), AST, ALT, LDH and GGT enzymes activity. Our data suggested clearly that the elevated liver enzymes could be a sign of oxidative stress-dependent disruption of the hepatocyte's lipid bilayer. The lipid bilayer is made up of polyunsaturated fatty acids, oxidation of which could result in membrane disruption with subsequent escape of detectable quantity of hepatic enzymes (32). Interestingly, vitamin $\mathrm{C}$ (ascorbic acid) countered ciprofloxacin-dependent effects in a mice model of ALF most probably via an antioxidant mechanism
(33). The antioxidant capacity of vitamin $\mathrm{C}$ is well documented in the liver as well as other tissues $(18,33)$. We concluded then that ciprofloxacininduced oxidative stress and its resultant hepatic damage were countered by restoring normal liver GSH storage level. The restoration of normal GGT activity rationally supported this assumption. Supporting our idea, GSH was more effective than $\mathrm{N}$-Acetyl-Cystein in the treatment of acetaminophen (APAP)-induced ALF in mice. This was probably due to the higher amino acids content which could be utilized for the resynthesize of hepatic GSH itself, as well as an energy substrates in the Krebs cycle (17).

In an attempt to elucidate possible underlying mechanisms, we studied changes that may have occurred on the mRNA level. Our results demonstrated that the mRNA expression levels of CYP3A, and the protective antioxidants genes GPx, GSR and Nrf2 were significantly downregulated in the ciprofloxacin treated group when compared to the corresponding values in the control group. Ciprofloxacin has been reported to reduce hepatic CYP3A expression (34).The down regulation of CYP3A in response to ciprofloxacin treatment could be explained by the exhaustion of the enzyme in response to the overwhelming drug metabolism. Ciprofloxacin-induced generation of free radicals frequently occurs during either direct redox cycling of the drug or its oxidative metabolism by CYP3A (35). Accordingly, the possible poor coupling of CYP3A catalytic cycle may have resulted in incessant production of ROS. During biotransformation, ciprofloxacin could be converted to quinone metabolites. Consequently, this could lead the formation of GSH adducts, depletion of the antioxidants pool and generation 
of excessive ROS (36). Raising the possibility of viscous cycle formation, free radicals can inhibit CYP3A activity via heme degradation (37). CYP3A-mediated metabolism under GSH depletion conditions aggravated ALF (38). Generation of ROS would possibly have overwhelmed the innate antioxidant defences, particularly and of our interest GSH. The activity of GPx and GSR reached the maximum level at first 96 hours in a rat model of tetrachloromethane (CCL4)-induced ALF. GPx and GSR activities significantly declined afterwards (31). These pro-oxidizing cellular conditions could in turn cause lipid peroxidation, DNA and protein damage, interruption altered gene expression or of signalling pathways (19). The antioxidant response element (ARE)-mediated transcription of the genes involved in the regulation of both the synthesis and conjugation of glutathione is controlled by the master transcription factor Nrf2 (39). Nrf2 also regulates the gene expression of GPx and GSR $(40,41)$. It wasn't a surprise then that Nrf2 mRNA level was also down-regulated in the ciprofloxacin treated group. Restoration of the hepatic GSH level was then a sensible strategy. Firstly, GSH is the most abundant cellular antioxidant, and secondly GSH is primarily synthesized in liver and plays vital roles in detoxification and antioxidant systems. Moreover, GSH has been used to treat acute and chronic liver diseases (42). In the present study, glutathione treatment was associated with significant upregulation of CYP3A, GPx, GSR and Nrf2 genes expression levels. It was reported previously that treatment with S-ally glutathione (SAG) resulted in significant increase in GPx activity in CCL4- induced ALF in rats. Not only SAG has quenched the oxidative stress markers, it also preserved the endogenous antioxidants including GSH (27). GPx itself reduces lipid hydroperoxides which require GSH, thus increasing GPx activity will in turn preserve the GSH stores. The up-regulation of Nrf2 gene by GSH therapy played an important role in the restoration of the liver functions in our study. Exogenous GSH was able to increase the activity Nrf2 and protected RAW 264.7 cells against oxidative stress-induced mitochondriamediated apoptosis via with the activity of the Nrf2/HO-1 mediated pathway (43). Convincingly, we proposed that GSH increased Nrf2 expression. The up-regulated Nrf2 increased the expression of GPx and GSR which in turn preserved the GSH cellular content. The CYP3A results indicate restoration of hepatic metabolic capabilities was achieved following alleviation of the pro-oxidative condition by GSH.

\section{CONCLUSION}

Glutathione could serve as a potential therapeutic molecule for liver diseases. Exogenous glutathione normalized the liver functions enzymes; ALT, AST, GGT and LDH, possibly by protecting hepatocytes membranes from disruption by oxidizing metabolites. Moreover, glutathione had gene modifying potentials on the antioxidant genes GPx, GSR and Nrf2, as well as preservation of CYP3A activity denoting the restoration of hepatic metabolic capabilities oxidative stress conditions.

\section{ABBREVIATIONS}

Acute liver failure (ALF), acetaminophen (APAP), acid phosphatase (ACP), alanine aminotransferase (ALT), antioxidant response element (ARE), aspartate aminotransferase (AST), The cytochrome P450 (CYP), cytochrome P450-family 3- 
subfamily A (CYP3A), glutathione (GSH), glutathione reductase (GSR), glutathione Stransferase (GST), glyceraldehyde 3-phosphate dehydrogenase (GAPDH), nuclear factor erythroid 2 (NFE2)-related factor (Nrf2), reactive oxygen species (ROS), reverse transcriptionpolymerase chain reaction (RT-PCR), tumor necrosis factor (TNF)- $\alpha$.

\section{DECLARATIONS}

ETHICS APPROVAL AND CONSENT TO PARTICIPATE

This study was approved by the Ethical Committee of the Faculty of Medicine, Menoufia University, Egypt.

\section{CONSENT TO PUBLISH}

Not applicable.

\section{AVAILABILTY OF DATA AND MATERIALS}

Data supporting findings are presented within the manuscript.

\section{COMPETING INTERESTS}

No conflict of interests.

\section{FUNDING}

This research did not receive any specific grant from funding agencies in the public, commercial, or not-for-profit sectors.

\section{AUTHORS' CONTRIBUTIONS}

YMN carried out the PCR experiments, participated in the study design and coordination, analysed the results, performed the statistical analysis and drafted the manuscript. SMW and OA carried out the animal experiments and biochemical assays, and participated in the study design. All authors have read and approved the final version of the manuscript.

\section{AKNOWLEGMENTS}

Authors would also like to acknowledge the assistance of Prof. Dr. Eman Badr and all members of the Central Lab - Faculty of Medicine Menoufia University.

\section{REFRENCES}

1. Pathikonda M, Munoz SJ. Acute Liver Failure. Annals of Hepatology. 2010;9(1):714.

\section{Bernal W, Donaldson N, Wyncoll D, Wendon}

J. Blood lactate as an early predictor of outcome in paracetamol-induced acute liver failure: a cohort study. Lancet (London, England). 2002;359(9306):558-63.

3. Katarey D, Verma S. Drug-induced liver injury. Clinical medicine (London, England). 2016;16(Suppl 6):s104-s9.

\section{Sanchez-Valle V, Chavez-Tapia NC, Uribe}

M, Mendez-Sanchez N. Role of oxidative stress and molecular changes in liver fibrosis: a review. Current medicinal chemistry. 2012;19(28):4850-60.

5. Cichoz-Lach H, Michalak A. Oxidative stress as a crucial factor in liver diseases. World journal of gastroenterology. 2014;20(25):8082-91.

6. Singal AK, Jampana SC, Weinman SA. Antioxidants as therapeutic agents for liver disease. Liver international : official journal of the International Association for the Study of the Liver. 2011;31(10):1432-48.

\section{Pham TDM, Ziora ZM, Blaskovich MAT.} Quinolone antibiotics. MedChemComm. 2019;10(10):1719-39. 
8. Qutrio Baloch Z, Raza MA, Abbas SA, Bukhari S. Ciprofloxacin-induced Hepatotoxicity in a Healthy Young Adult. Cureus. 2017;9(2):e1016.

9. Giannini EG, Testa R, Savarino V. Liver enzyme alteration: a guide for clinicians. CMAJ : Canadian Medical Association journal $=$ journal de l'Association medicale canadienne. 2005;172(3):367-79.

10. Talla V, Veerareddy P. Oxidative stress induced by fluoroquinolones on treatment for complicated urinary tract infections in Indian patients. Journal of young pharmacists : JYP. 2011;3(4):304-9.

11. Li S, Tan H-Y, Wang N, Zhang Z-J, Lao L, Wong C-W, et al. The Role of Oxidative Stress and Antioxidants in Liver Diseases. International journal of molecular sciences. 2015;16(11):26087-124.

12. Birben E, Sahiner UM, Sackesen C, Erzurum S, Kalayci O. Oxidative stress and antioxidant defense. The World Allergy Organization journal. 2012;5(1):9-19.

13. Bocedi A, Noce A, Marrone G, Noce G, Cattani G, Gambardella G, et al. Glutathione Transferase P1-1 an Enzyme Useful in Biomedicine and as Biomarker in Clinical Practice and in Environmental Pollution. Nutrients. 2019;11(8):1741.

\section{Chen Y, Dong H, Thompson DC, Shertzer} HG, Nebert DW, Vasiliou V. Glutathione defense mechanism in liver injury: insights from animal models. Food Chem Toxicol. 2013;60:38-44.

15. Yuan L, Kaplowitz N. Glutathione in liver diseases and hepatotoxicity. Molecular Aspects of Medicine. 2009;30(1):29-41.
16. Hayward KL, Powell EE, Irvine KM, Martin JH. Can paracetamol (acetaminophen) be administered to patients with liver impairment? British journal of clinical pharmacology. 2016;81(2):210-22.

17. Saito C, Zwingmann C, Jaeschke H. Novel mechanisms of protection against acetaminophen hepatotoxicity in mice by glutathione and N-acetylcysteine. Hepatology (Baltimore, Md). 2010;51(1):246-54.

18. Rizk NI, Rizk MS, Mohamed AS, Naguib YM. Attenuation of sleep deprivation dependent deterioration in male fertility parameters by vitamin C. Reprod Biol Endocrinol. 2020;18(1):2.

19. Sant KE, Hansen JM, Williams LM, Tran NL, Goldstone JV, Stegeman JJ, et al. The role of Nrf1 and Nrf2 in the regulation of glutathione and redox dynamics in the developing zebrafish embryo. Redox Biology. 2017;13:207-18.

20. Zanger UM, Schwab M. Cytochrome P450 enzymes in drug metabolism: Regulation of gene expression, enzyme activities, and impact of genetic variation. Pharmacology \& Therapeutics. 2013;138(1):103-41.

21. Phaniendra A, Jestadi DB, Periyasamy L. Free radicals: properties, sources, targets, and their implication in various diseases. Indian journal of clinical biochemistry : IJCB. 2015;30(1):11-26.

22. Ameen O, Yassien RI, Naguib YM. Activation of FoxO1/SIRT1/RANKL/OPG pathway may underlie the therapeutic effects of resveratrol on aging-dependent male osteoporosis. BMC musculoskeletal disorders. 2020;21(1):375. 
23. El Agamy DF, Naguib YM. CoQ10 ameliorates monosodium glutamate-induced alteration in detrusor activity and responsiveness in rats via antiinflammatory, anti-oxidant and channel inhibiting mechanisms. BMC Urol. 2019;19(1):103.

24. Motawea SM, Noreldin RI, Naguib YM. Potential therapeutic effects of endothelial cells trans-differentiated from Wharton's Jelly-derived mesenchymal stem cells on altered vascular functions in aged diabetic rat model. Diabetology \& metabolic syndrome. 2020;12:40.

25. Chalasani $\mathbf{N}, \mathbf{B} \mathbf{j} \tilde{A} \rrbracket$ rnsson E. Risk factors for idiosyncratic drug-induced liver injury. Gastroenterology. 2010;138(7):2246-59.

\section{Naguib YM, Azmy RM, Samaka RM, Salem}

MF. Pleurotus ostreatus opposes mitochondrial dysfunction and oxidative stress in acetaminophen-induced hepatorenal injury. BMC complementary and alternative medicine. 2014;14:494.

27. Thayumanavan P, Loganathan C, Iruthayaraj A, Poomani K, Nallaiyan S. S-allyl-glutathione, a synthetic analogue of glutathione protected liver against carbon tetrachloride toxicity: Focus towards antioxidative efficiency. Environmental Toxicology and Pharmacology. 2018;58:218.

\section{McDowell Torres D, Stevens RD, Gurakar}

A. Acute liver failure: a management challenge for the practicing gastroenterologist. Gastroenterology \& hepatology. 2010;6(7):444-50.

29. Zhang H, Forman HJ. Redox regulation of gamma-glutamyl transpeptidase. American journal of respiratory cell and molecular biology. 2009;41(5):509-15.

30. Kotoh K, Kato M, Kohjima M, Tanaka M, Miyazaki M, Nakamura K, et al. Lactate dehydrogenase production in hepatocytes is increased at an early stage of acute liver failure. Experimental and therapeutic medicine. 2011;2(2):195-9.

31.Iskusnykh IY, Popova TN, Agarkov AA, Pinheiro de Carvalho MÃA, Rjevskiy SG. Expression of Glutathione Peroxidase and Glutathione Reductase and Level of Free Radical Processes under Toxic Hepatitis in Rats. Journal of toxicology. 2013;2013:870628-

32. Choudhary AK, Devi RS. Serum biochemical responses under oxidative stress of aspartame in wistar albino rats. Asian Pacific Journal of Tropical Disease. 2014;4:S403-S10.

33. Weyers AI, Ugnia LI, GarcÃ-a Ovando H, Gorla NB. Ciprofloxacin increases hepatic and renal lipid hydroperoxides levels in mice. Biocell : official journal of the Sociedades Latinoamericanas de Microscopia Electronica et al. 2002;26(2):225-8.

34. Toda T, Ohi K, Kudo T, Yoshida T, Ikarashi N, Ito $\mathbf{K}$, et al. Ciprofloxacin Suppresses Сyp3a in Mouse Liver by Reducing Lithocholic Acid-producing Intestinal Flora. Drug Metabolism and Pharmacokinetics. 2009;24(3):201-8.

35. Veith A, Moorthy B. ROLE OF CYTOCHROME P450S IN THE GENERATION AND METABOLISM OF REACTIVE OXYGEN SPECIES. Current opinion in toxicology. 2018;7:44-51. 
36. Deavall DG, Martin EA, Horner JM, Roberts R. Drug-induced oxidative stress and toxicity. Journal of toxicology. 2012;2012:645460-.

37. Albertolle ME, Peter Guengerich F. The relationships between cytochromes P450 and $\mathrm{H} 2 \mathrm{O} 2$ : Production, reaction, and inhibition. Journal of Inorganic Biochemistry. 2018;186:228-34.

38. Jiang L-L, Jiang Y, Zhao D-S, Fan Y-X, Yu $\mathbf{Q}$, Li P, et al. CYP3A Activation and Glutathione Depletion Aggravate EmodinInduced Liver Injury. Chemical Research in Toxicology. 2018;31(10):1052-60.

39. Yamashita $\mathbf{Y}$, Ueyama $\mathbf{T}$, Nishi $\mathbf{T}$, Yamamoto Y, Kawakoshi A, Sunami S, et al. Nrf2-inducing anti-oxidation stress response in the rat liver--new beneficial effect of lansoprazole. PloS one. 2014;9(5):e97419-e.

40. Gallorini M, Petzel C, Bolay C, Hiller K-A, Cataldi A, Buchalla W, et al. Activation of the Nrf2-regulated antioxidant cell response inhibits HEMA-induced oxidative stress and supports cell viability. Biomaterials. 2015;56:114-28.

41. Harvey CJ, Thimmulappa RK, Singh A, Blake DJ, Ling G, Wakabayashi N, et al. Nrf2-regulated glutathione recycling independent of biosynthesis is critical for cell survival during oxidative stress. Free radical biology \& medicine. 2009;46(4):443-53.

42. Honda Y, Kessoku T, Sumida Y, Kobayashi T, Kato T, Ogawa Y, et al. Efficacy of glutathione for the treatment of nonalcoholic fatty liver disease: an open-label, single- arm, multicenter, pilot study. BMC gastroenterology. 2017;17(1):96-.

43. Kwon DH, Cha HJ, Lee H, Hong SH, Park C, Park SH, et al. Protective Effect of Glutathione against Oxidative Stressinduced Cytotoxicity in RAW 264.7 Macrophages through Activating the Nuclear Factor Erythroid 2-Related Factor2/Heme Oxygenase-1 Pathway. Antioxidants (Basel, Switzerland). 2019;8(4). 